\section{Modified Rendezvous Technique for Bilateral Biliary Drainage Through a Jejunal Interponat of the Common Bile Duct with Anastomotic Strictures}
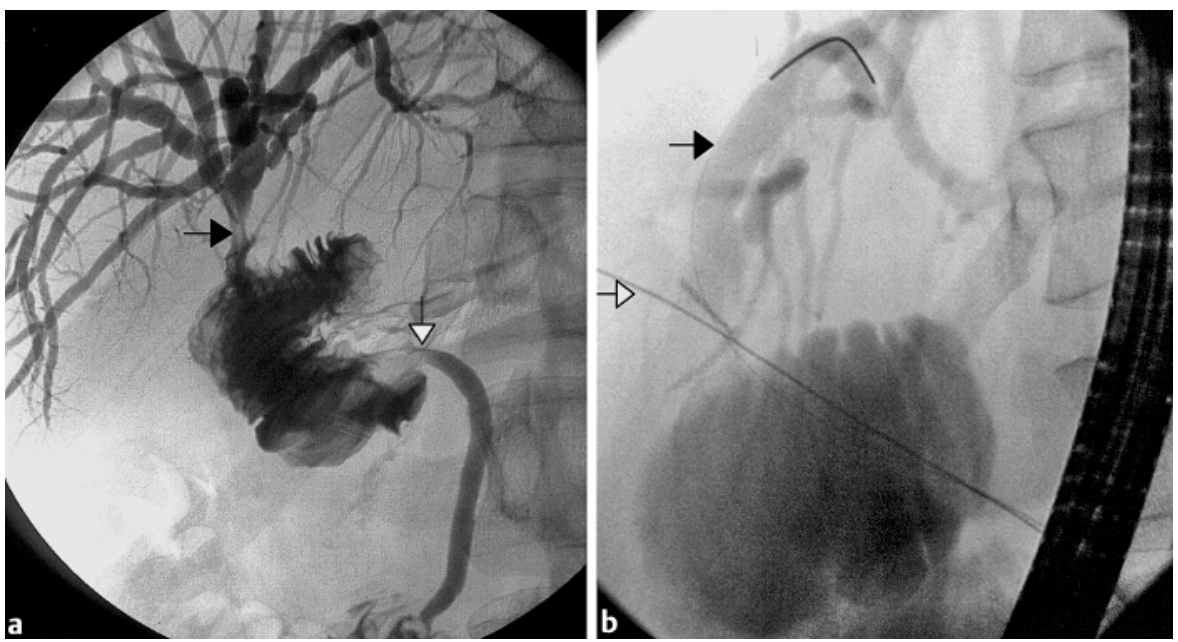

Figure 1 Modified rendezvous technique for bilateral biliary drainage in a patient with a jejunal interponat of the common bile duct and anastomotic strictures. a Percutaneous trans-hepatic cholangiography showing the jejunal interponat with moderate stenosis of the hepatojejunal anastomosis (black arrow) and of the jejuno-biliary anastomosis (white arrow). $\mathbf{b}$ The percutaneously introduced wire (white arrow) has been grasped with a Dormia basket in the jejunal interponat and pulled through the endoscope. Using an 8.5-Fr pushing catheter placed over the first wire through the endoscope, a second wire (black arrow) was placed in the left intrahepatic bile duct. c The final result, showing two 8.5-Fr, 15-cm endoprostheses, one in the left intrahepatic bile duct and one in the right intrahepatic bile duct, and an $8.5-\mathrm{Fr}, 9-\mathrm{cm}$ endoprosthesis in the jejunal interponat.

The proximal common bile duct was accidentally removed during laparoscopic cholecystectomy in a 43-year-old man. Biliary drainage was successfully re-established in our clinic by the formation of a jejunal interponat, but the patient's cholestasis relapsed, due to delayed biliary drainage and moderate stenosis of both jejunal anastomoses (Figure 1a). Attempts to manage this by crossing of the interponat to the opposite opening with a guide-wire during endoscopic retrograde cholangiography or percutaneous trans-hepatic cholangiography failed.

A rendezvous maneuver was then performed. First, a guide-wire was advanced into the interponat via percutaneous trans-hepatic cholangiography, where it was grasped with a Dormia basket and was used to guide an 8.5-Fr pushing catheter (PC 8.5; Wilson-Cook, WinstonSalem, North Carolina, USA) through the endoscope to the proximal anastomosis. A second guide-wire was then inserted through this catheter into the left intra- hepatic bile duct (Figure $\mathbf{1 b}$ ). With two guide-wires in place, an $8.5-\mathrm{Fr}, 15-\mathrm{cm}$ endoprosthesis was advanced, after dilation, into each hepatic lobe. Finally, a third transpapillary, 8.5- $\mathrm{Fr}$, 9-cm endoprosthesis was placed into the jejunal interponat (Figure 1 c). The patient's cholestasis resolved and 6 months later no significant strictures were detected, and all the endoprostheses were removed. The patient has been asymptomatic for 4 years.

In this case, a rendezvous maneuver [1] was combined with a double-wire technique using a pushing catheter for the insertion of a second guide-wire through a biliary jejunal interponat into the left lobe of the liver. Bilateral hepatic drainage was thus ensured without the need for a second percutaneous puncture, and dilation of the strictures with placement of endoprostheses resulted in sustained remission of the patient's cholestasis, even after removal of the stents.

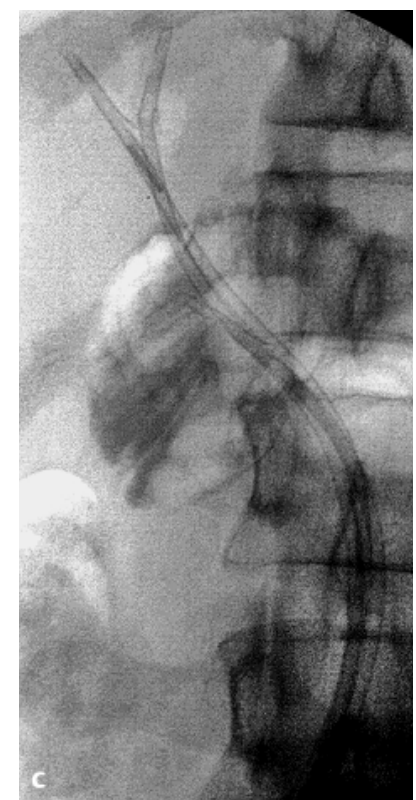

U. Töx ${ }^{1}$, H.-M. Steffen ${ }^{1}$, K. Lackner ${ }^{2}$, A. H. Hölscher ${ }^{3}$, T. Goeser ${ }^{1}$

${ }^{1}$ Department of Gastroenterology, University of Cologne, Cologne, Germany

2 Department of Radiology, University of Cologne, Cologne, Germany

${ }^{3}$ Department of Surgery, University of Cologne, Cologne, Germany

\section{Reference}

${ }^{1}$ Sommer A, Burlefinger R, Bayerdorffer E et al. Internal biliary drainage in the "rendezvous" procedure. Combined transhepatic endoscopic retrograde methods. Dtsch Med Wochenschr 1987; 112: 747-775

\section{Correspondling Author}

\section{U. Töx, M.D.}

Klinik IV Innere Medizin,

Schwerpunkt Gastroenterologie und Hepatologie, Klinikum der Universität zu Köln

50924 Köln

Germany

Fax: $\quad+49-221-4786758$

E-mail: ulrich.toex@medizin.uni-koeln.de 